\title{
La radioprotection sur EPR : présentation comparée des instructions françaises et finlandaises et des démarches d'optimisation à la conception
}

E. ARIAL ${ }^{1}$, O. COUASNON ${ }^{1}$, N. LATIL-QUERREC ${ }^{1}$, J.M. EVRARD ${ }^{2}$, K. HERVIOU ${ }^{3}$, V. RIIHILUOMA ${ }^{4}$, Y. BENETEAU ${ }^{5}$, J.L. FORET ${ }^{5}$

(Manuscrit reçu le 26 janvier 2010, accepté le 18 mai 2010)

RÉSUMÉ À l'occasion de l'évaluation du rapport préliminaire de sûreté du réacteur EPR de Flamanville 3 (France), l'IRSN a souhaité dresser un bilan de l'historique de l'EPR, depuis les années 1990 jusqu'à l'autorisation de construction d'un réacteur EPR en Finlande puis en France ainsi que des instructions menées sur les aspects liés à la prise en compte de la radioprotection des travailleurs. Ce bilan présente les objectifs de dose retenus par les exploitants à la conception, la démarche globale d'optimisation mise en œuvre ainsi qu'une comparaison des démarches d'analyse française et finlandaise. En France, par exemple, EDF a réalisé des études approfondies d'optimisation sur les activités à fort enjeu « radioprotection » (calorifuge, logistique de chantier, robinetterie, ouverture/fermeture de la cuve, générateur de vapeur, évacuation du combustible et conditionnement des déchets). Cette démarche repose (en France) sur un processus itératif d'optimisation. Une comparaison des objectifs de dose collective des EPR français et finlandais par rapport aux doses reçues sur d'autres réacteurs à eau sous pression (Konvoi allemands, tranches du parc français, ....) est également présentée. Cette synthèse, élaborée par l'IRSN en collaboration avec EDF et l'autorité de sûreté nucléaire finlandaise STUK, reflète plus de $\mathbf{1 5}$ ans d'études et de partenariat en matière de radioprotection, dans le cadre du projet EPR.

ABSTRACT Radiation protection on EPR: comparative approach of the French and Finnish regulatory reviewing process and optimization at the design phase.

Taking the opportunity to evaluate the preliminary safety report of the French EPR reactor built in Flamanville, the IRSN proposes to assess the history of EPR, from the decision to implement studies in the 90s to the French and German cooperation, and finally to the construction of a unit in Finland and in France, and to make a synthesis of the assessment of radiation protection arrangements. This assessment presents the dose targets (calculated reference doses) planned by the nuclear operators in the design phase as well as the global radiation protection optimization process and a comparison of French and Finnish analyses. In France, for example,

IRSN, Unité d'Expertise en radioprotection des travailleurs et de la population, DRPH/SER/UETP, BP 17, 92262 Fontenay-aux-Roses, France.

2 IRSN, Direction scientifique, DS, BP 17, 92262 Fontenay-aux-Roses, France.

IRSN, Direction de la sûreté des réacteurs, DSR, BP 17, 92262 Fontenay-aux-Roses, France.

4 Radiation and Nuclear Safety Authority (STUK), Laippatie 4, PO Box 14, 00881 Helsinki, Finlande.

5 Électricité de France-CNEN (EDF), 165 avenue Pierre Brossolette, BP 900, 92542 Montrouge Cedex, France. 


\begin{abstract}
EDF performed a detailed optimization analysis of selected tasks known to have a major contribution to the annual average collective dose (thermal insulation, logistics, valve maintenance, opening/closing of the vessel, preparation and checks of steam generators, on-site spent fuel management, and waste management). The optimization process is based (in France) on an iterative method. A comparison between the EPR collective dose target and doses received in other pressurized water reactors that are close to the EPR design (Konvoi of German design, French existing units, etc.) is also presented. This synthesis was carried out by the IRSN, the expert body of the French nuclear safety authority, in association with Electricité de France (EDF), the French operator, and the authority for nuclear safety in Finland (STUK). It summarizes more than $\mathbf{1 5}$ years of studies and partnership, focusing on radiation protection, in the design phase of the EPR.
\end{abstract}

Keywords: EPR / radiation protection / optimization

\title{
Introduction
}

Deux réacteurs de type $\mathrm{EPR}^{6}$ (European Pressurized water Reactor) sont actuellement en construction en Europe, l'un en Finlande (Fig. 1) sur le site d'Olkiluoto (OL3), le second en France (Fig. 2) sur le site de Flamanville (FLA3). L'Autorité de sûreté nucléaire (ASN) a sollicité l'Institut de radioprotection et de sûreté nucléaire (IRSN), son appui technique, pour mener l'instruction du rapport de sûreté de l'EPR français. Les aspects relatifs à la prise en compte de la radioprotection à la conception sont notamment analysés dans ce cadre.

Depuis 2007, l'IRSN a entrepris de réaliser, en collaboration avec l'autorité de sûreté nucléaire finlandaise (STUK) et l'exploitant nucléaire français EDF, une comparaison synthétique des deux systèmes d'instruction français et finlandais ainsi que des objectifs de dose retenus et des démarches d'optimisation à la conception mises en œuvre par les deux maîtres d'ouvrages et exploitants concernés ${ }^{7}$. La synthèse est sensiblement plus détaillée pour la France du fait de la participation directe d'EDF à cet article et elle s'intéresse spécifiquement aux actions menées à partir de 2004 (à partir de cette date la démarche d'optimisation s'est nettement structurée en prenant en compte le retour d'expérience des meilleures tranches du parc, l'efficacité des options a été quantifiée et un objectif de dose collective correspondant aux ambitions de l'EPR a été élaboré).

\footnotetext{
6 À noter que l'appellation «EPR» est devenue depuis peu, à l'initiative d'AREVA, une marque de commerce non enregistrée : EPR ${ }^{\mathrm{TM}}$. Par le signe TM (pour « trade-mark») qui accompagne l'appellation, AREVA indique publiquement sa volonté d'empêcher l'emploi de toute autre marque semblable par des concurrents. La portée juridique du signe TM est très variable d'un pays à l'autre (aucune portée juridique en France par exemple) et les initiales relatives aux termes génériques et descriptifs du European Pressurized water Reactor, très employées depuis le partenariat franco-allemand, restent bien sûr largement utilisées en dehors des relations commerciales.

7 Les objectifs imposés par les maitres d'ouvrages dans les contrats d'études et de fournitures ne sont pas l'objet de cet article. Le point de vue et la contribution du concepteur AREVA aux études d'optimisation du projet ne sont notamment pas traités dans cet article, bien que ce dernier joue un rôle majeur dans l'optimisation de la conception des EPR.
} 
Les objectifs de dose définis pour les deux réacteurs EPR sont également comparés aux doses reçues sur différents réacteurs actuellement en fonctionnement en France et en Allemagne.

Après un bref rappel de l'historique, cet article présente les principaux éléments de comparaison issus de ces analyses (Gourram et al., 2008 ; Couasnon et al., 2008 ; Bouchez et al., 2009).

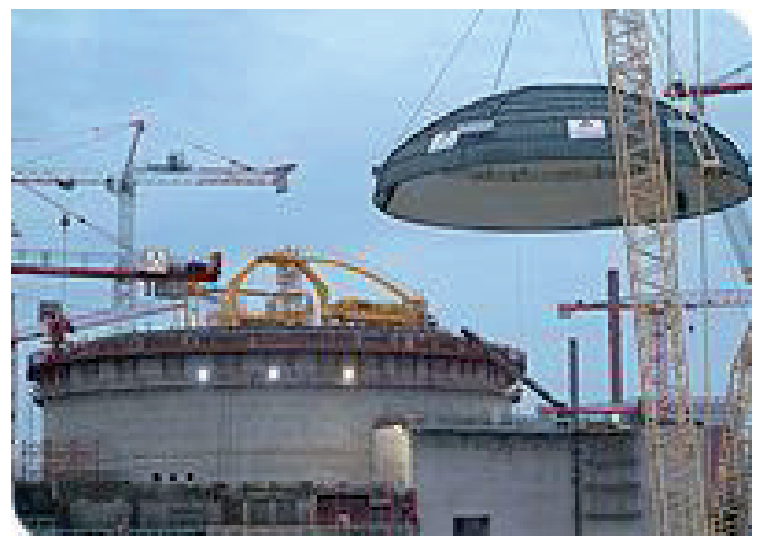

Figure 1 - Chantier de l'EPR finlandais d'Olkiluoto (septembre 2009).

Olkiluoto construction site of the Finnish EPR (September 2009).

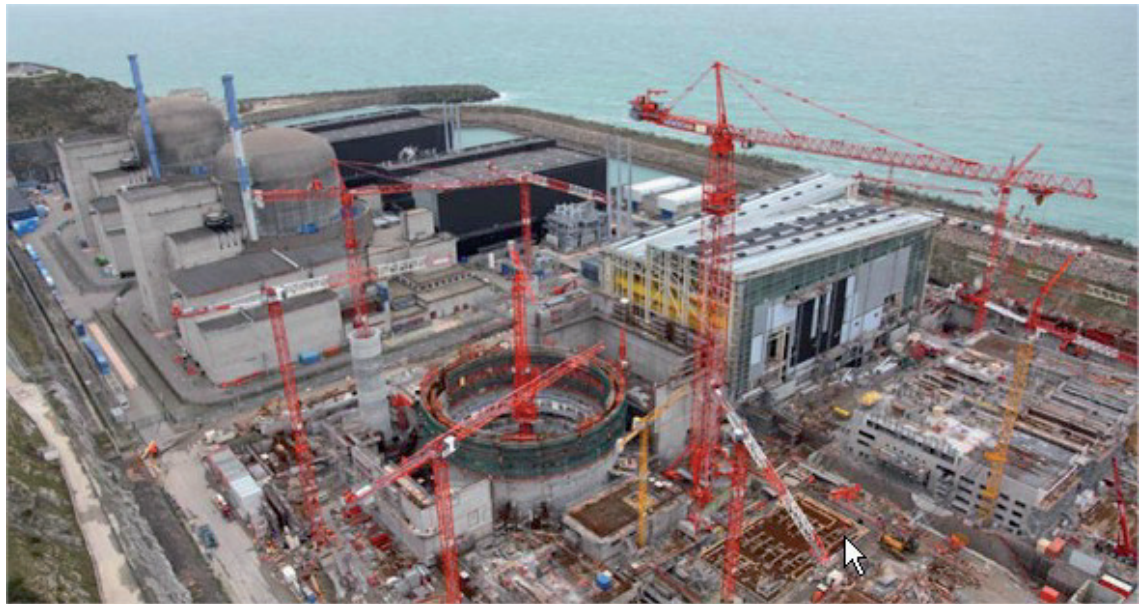

Figure 2 - Chantier de l'EPR français de Flamanville (décembre 2009).

Flamanville construction site of the first French EPR (December 2009). 


\section{Historique}

À la fin des années 80 , des réflexions sur la sûreté d'éventuels futurs réacteurs nucléaires ont débuté en France puis ont été poursuivies par la mise en œuvre d'un partenariat entre l'IRSN et son homologue allemand (GRS : Gesellschaft für Anlagen und Reaktorsicherheit). Les discussions menées entre les deux organismes les ont conduits à formuler, à la demande des autorités de sûreté des deux pays, des propositions communes concernant la démarche et les objectifs généraux de sûreté et de radioprotection à retenir pour les futurs réacteurs nucléaires à eau sous pression. Ces objectifs de sûreté ont été approuvés par les deux autorités en 1993 (DSIN, 1993). Par ailleurs, le projet EPR était mis en place par l'industrie, associant l'exploitant français EDF, un consortium d'électriciens allemands et la société NPI, filiale de Framatome et de Siemens. Dans ce cadre, des propositions d'options générales de conception visant à satisfaire les objectifs généraux de sûreté retenus ont permis de préciser progressivement le projet EPR. Les partenaires (Framatome, Siemens, NPI, Électricité de France et neuf électriciens allemands) ont soumis officiellement aux autorités de sûreté française et allemande, en octobre 1997, un rapport appelé «Basic Design Report » présentant le résultat de l'avant-projet détaillé.

En 1998, la décision de l'Allemagne d'abandonner le développement de l'énergie nucléaire met fin au partenariat entre la France et l'Allemagne sur le projet.

Le groupe permanent pour les réacteurs nucléaires (qui constitue un des appuis techniques de l'autorité de sûreté en France), auquel des experts allemands étaient encore associés, a approuvé en octobre 2000 un document intitulé «Technical Guidelines for the Design and the Construction of the Next Generation of Nuclear Power Plants with Pressurized Water Reactors », sur la base d'une proposition élaborée par l'IRSN et GRS. Ce document, après avoir rappelé les objectifs généraux de sûreté définis en 1993, tenait compte des discussions menées avec le projet EPR de 1993 à 2000. Il précisait les objectifs généraux de sûreté et mentionnait les dispositions et les conditions acceptables pour y répondre (objectif de réduction des doses individuelles et collectives en phase d'exploitation normale et incidentelle, optimisation de ces doses, choix des matériaux, fiabilité des composants, robotique, opérations de maintenance tenant compte du retour d'expérience, ...).

En 2002, une décision de principe de construire un nouveau réacteur en Finlande, basé sur la technologie EPR, a été prise ( $c f$. Sect. 2.2 qui détaille les différentes étapes du processus mené en Finlande). Le site d'Olkiluoto a été sélectionné par l'opérateur TVO (Teollisuuden Voima Oyj) et un contrat avec le 
consortium AREVA (Framatome ANP) - Siemens a été signé en décembre 2003. Une nouvelle coopération, cette fois entre la France et la Finlande, a débuté et des échanges techniques ont lieu régulièrement entre l'autorité de sûreté finlandaise (STUK) et l'autorité de sûreté française (ASN) ainsi que son appui technique, l'IRSN.

Le Preliminary Safety Analysis Report (PSAR) de l'EPR OL3 a été soumis début 2004 à l'autorité de sûreté finlandaise. En France, EDF a également soumis un PSAR dit «édition 2003 » à cette même période (la première version projet d'un PSAR générique avait été transmise dès 2001).

L'autorisation de construction de l'EPR finlandais a été délivrée en 2005 par le gouvernement. La France a elle-même engagé la construction d'un réacteur de type EPR suite au dépôt de la demande d'autorisation de création de cette installation sur le site de Flamanville en mai 2006 par EDF. Le décret d'autorisation de création a été signé en avril 2007 par le gouvernement.

Suite à des retards sur la réalisation du chantier, la mise en service industrielle de l'EPR finlandais, initialement prévue en 2009, a été reportée à 2012. En ce qui concerne le projet français, la mise en service est actuellement toujours programmée en 2012 mais risque également de glisser au-delà.

La figure 3 présente les grandes étapes de l'historique de l'EPR.
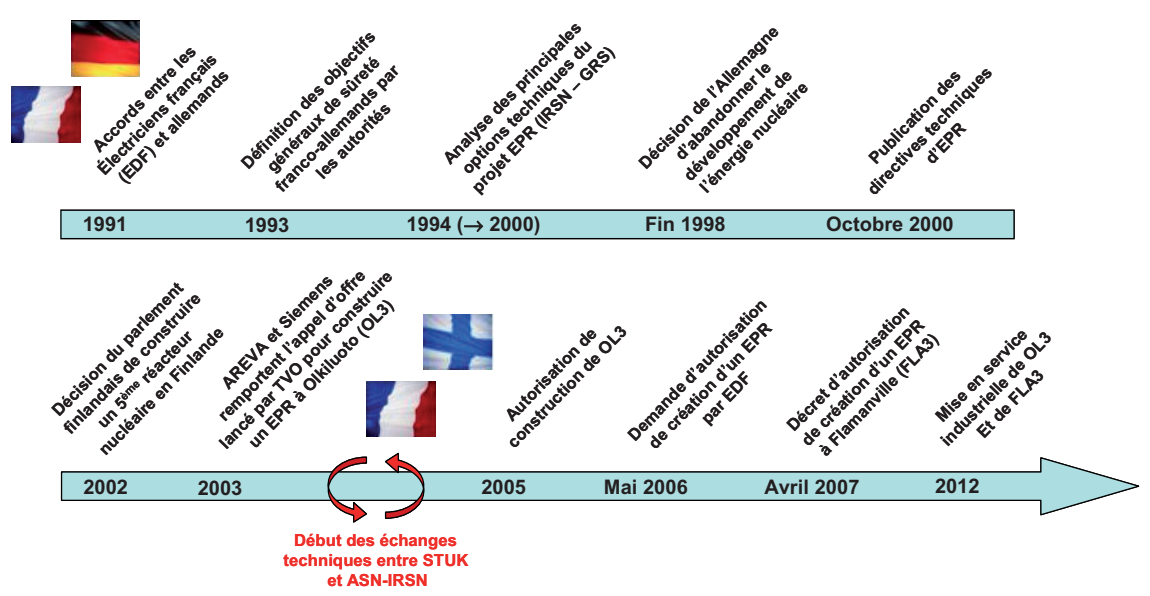

Figure 3 - Partenariat entre la France et l'Allemagne puis avec la Finlande.

French-German cooperation and French-Finnish cooperation. 


\section{L'instruction des dossiers}

Que ce soit en France ou en Finlande, la mise en œuvre d'un projet de nouveau réacteur nucléaire commence par un dépôt de dossier par l'exploitant. Ce dossier est ensuite évalué par l'autorité de chaque pays selon la réglementation nationale en vigueur.

\subsection{Instruction en France}

En France, les principales parties prenantes impliquées dans le système d'instruction sont :

- l'Autorité de sûreté nucléaire : ASN ;

- les appuis techniques de l'autorité :

- l'Institut de radioprotection et de sûreté nucléaire (IRSN) en charge de l'expertise dans les domaines de la sûreté et de la radioprotection ;

- le groupe permanent réacteurs (GPR) qui fournit ses recommandations à l'ASN sur la base des avis techniques de l'IRSN ;

- l'exploitant de l'EPR Flamanville 3 : Électricité de France (EDF).

Le processus d'autorisation global comporte plusieurs grandes étapes, allant de la prise de position à caractère technique de l'ASN sur les options de sûreté en date de 2004, au décret d'autorisation de création signé par le gouvernement en avril 2007, à l'obtention du permis de construire ${ }^{8}$, jusqu'à l'autorisation de mise en service, qui sera décidée par l'ASN.

Le processus d'autorisation de création et de mise en service est décrit succinctement sur la figure 4.

En préalable à la demande d' autorisation de création, EDF a saisi, conformément au code de l'environnement, la commission nationale du débat public sur le projet de construction. Un débat public a été organisé d'octobre 2005 à février 2006 sur

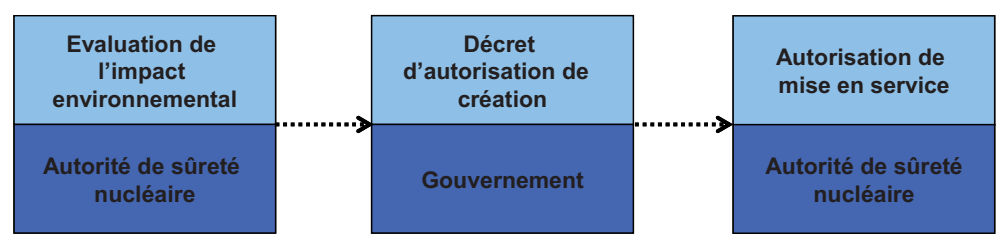

Figure 4 - Processus d'autorisation pour FLA3.

Licensing process of FLA3.

\footnotetext{
8 Les réacteurs électronucléaires sont soumis au régime général des permis de construire, et conformément au code de l'urbanisme ils nécessitent un permis délivré au nom de l'Etat. Dans ce cadre, la construction d'un EPR fait partie des « grands travaux » soumis à enquête publique.
} 
le territoire régional d'implantation de la future installation et sur le territoire national. La demande d'autorisation de création n'a été déposée qu'à l'issue des conclusions du débat public.

L'autorisation de création est accordée par décret pris sur le rapport des ministres chargés de la sûreté nucléaire. Ce dernier s'appuie sur l'avis du collège de l'ASN, lui-même établi sur la base d'un rapport de synthèse des services de l'ASN et de ses appuis techniques, l'IRSN et le GPR. La mise en service de l'installation doit avoir lieu au maximum 10 ans après la publication du décret d'autorisation de création (au-delà de 10 ans, la nouvelle réglementation s'appliquerait ${ }^{9}$ ). Le décret d'autorisation de création précise que l'introduction du combustible dans le périmètre de l'installation est soumise à l'autorisation de l'autorité de sûreté nucléaire sur la base d'un dossier comprenant le rapport de sûreté, les règles générales d'exploitation (RGE) et le plan d'urgence interne. Une réévaluation de la sûreté de l'installation est à prévoir tous les 10 ans. Le dossier relatif aux autorisations de rejets et de prélèvements d'eau fait l'objet d'une enquête publique et conduit à la publication d'un arrêté.

L'autorisation de mise en service, qui sera décidée par l'ASN, constitue la prochaine grande étape de l'instruction du dossier EPR Flamanville 3. Cette partie de l'instruction, portant sur l'analyse du rapport de sûreté de l'EPR Flamanville 3, est décrite de façon résumée sur la figure 5.

Afin de délivrer une autorisation de mise en service du réacteur de Flamanville 3, l'ASN sollicite l'IRSN pour évaluer notamment le contenu du rapport de sûreté et des règles générales d'exploitation, et le GPR pour donner son avis sur la base de l'instruction menée par l'IRSN. Après de nombreux échanges techniques avec l'exploitant, l'IRSN émet un rapport sur les conclusions de son

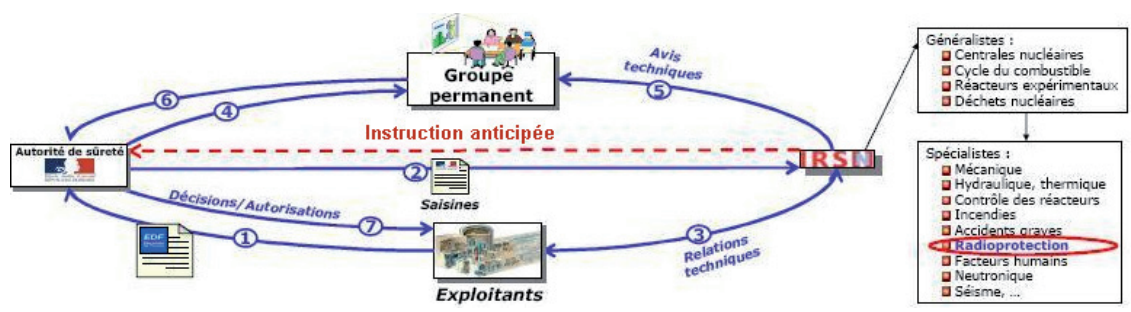

Figure 5 - Système d'instruction français. French reviewing process.

\footnotetext{
9 Il est à noter, qu'étant donné que la demande d'autorisation de création d'un EPR par EDF a été déposée en 2006, le décret a été délivré selon l'ancienne réglementation (décret $\mathrm{n}^{\circ}$ 63-1228 du 11 décembre 1963 relatif aux installations nucléaires). En effet, depuis le 2 novembre 2007, le décret $\mathrm{n}^{\circ}$ 2007-1557 fixe les procédures d'autorisation relatives aux installations nucléaires de base.
} 
instruction. Au terme de l'instruction de l'IRSN et d'une réunion contradictoire entre l'exploitant et l'IRSN, le GPR se prononce sur les points de désaccord subsistants. Le GPR donne ses conclusions à l'ASN qui prend la décision finale concernant l'autorisation de mise en service.

Dans le cas de l'EPR Flamanville 3, avant l'envoi complet du rapport provisoire de sûreté initialement prévu en octobre 2010, une instruction anticipée a été engagée sur la conception détaillée de l'installation, portant notamment sur la radioprotection et l'optimisation en phase de conception. Cette instruction anticipée est basée sur des dossiers techniques fournis par EDF.

En parallèle, pendant la phase de construction, l'ASN effectue des inspections afin de contrôler notamment la qualité de la réalisation et la conformité de l'installation aux études présentées dans le rapport de sûreté (8 inspections sur le chantier en 2007, 14 en 2008 et 24 en 2009).

En début d'année 2008, plusieurs inspections relatives aux opérations de génie civil ont permis à l'ASN de relever un manque de rigueur de l'exploitant et des prestataires externes dans la construction du futur réacteur et des insuffisances du système de management de la qualité d'EDF. Il est apparu notamment que la qualité du ferraillage du radier de l'îlot nucléaire n'était pas satisfaisante. Ce constat a conduit l'ASN à demander à EDF le 26 mai 2008 de suspendre ses travaux de bétonnage et de lui transmettre un plan d'actions correctives. L'arrêt des travaux a duré environ 3 semaines. Par ailleurs, en septembre 2008, l'ASN a mis en évidence des problèmes concernant les soudures du liner ${ }^{10}$. Les activités de fabrication ont été suspendues à la demande d'EDF compte-tenu de défauts de rigueur des contrôles exercés par le sous-traitant chargé de la fabrication du liner.

Sur la base des inspections menées, l'ASN a estimé à l'issue de l'année 2008 que la sûreté était prise en compte au sein du management du projet et des activités de construction mais que les écarts montraient que l'application des « exigences qualité » restait perfectible et que la culture de sûreté des différents intervenants du projet devait être améliorée. Enfin, parmi les inspections menées sur le chantier, certaines ciblent la radioprotection (opérations de gammagraphie).

\subsection{Instruction en Finlande}

En Finlande, les principales parties prenantes impliquées dans le processus d'instruction technique du rapport de sûreté sont :

- l'Autorité de sûreté nucléaire et de radioprotection: STUK (Säteilyturvakeskus) ;

${ }^{10}$ Liner : peau métallique assurant l'étanchéité de l'enceinte du réacteur. 
- les appuis techniques de l'autorité (TSO, Technical support organisations): différents organismes peuvent être sollicités en support de l'autorité (ex : le «Technical Research Centre »);

- l'exploitant du réacteur EPR OL3 situé à Olkiluoto: TVO (Teollisuuden Voima Oy).

Sous couvert du « Nuclear Energy Act» (1987), le ministère de l'emploi et de l'économie (ex-ministère du commerce et de l'industrie) est responsable des opérations concernant l'énergie nucléaire ainsi que la gestion des déchets. Il est assisté par un comité consultatif concernant l'énergie nucléaire sur différents sujets et également par un comité consultatif concernant la radioprotection.

L'autorité de sûreté nucléaire et de radioprotection (STUK) est responsable du contrôle et des inspections. L'autorité dépend du conseil d'État, qui délivre les autorisations de mise en service des installations nucléaires. STUK est sous la responsabilité du ministère des affaires sociales et de la santé et est assisté par le comité consultatif concernant la sûreté nucléaire.

Le «Nuclear Energy Act 990/1987 » et le décret 161/1988 définissent le processus d'autorisation dans son ensemble. La figure 6 présente le processus d'autorisation pour le réacteur OL3.

En mai 1998, le projet de construire un $5^{\mathrm{e}}$ réacteur nucléaire en Finlande démarre par une évaluation de l'impact environnemental (complétée en janvier 2000). En novembre 2000, le dossier de l'exploitant TVO est soumis à une décision de principe, prise en janvier 2002 par le gouvernement. En mai 2002, le parlement ratifie la décision et, par conséquent, TVO est autorisé à continuer les préparatifs en vue de la construction du nouveau réacteur. Le site d'Olkiluoto est sélectionné par l'exploitant TVO et un contrat avec le consortium AREVASiemens est signé en décembre 2003. En 2004, TVO envoie les dossiers de demande d'autorisation de construction au ministère de l'emploi et de l'environnement ainsi que le dossier technique à l'autorité de sûreté STUK (notamment le rapport préliminaire de sûreté). La mission du STUK est d'analyser la conception et la sûreté du réacteur afin de donner un avis au ministère de

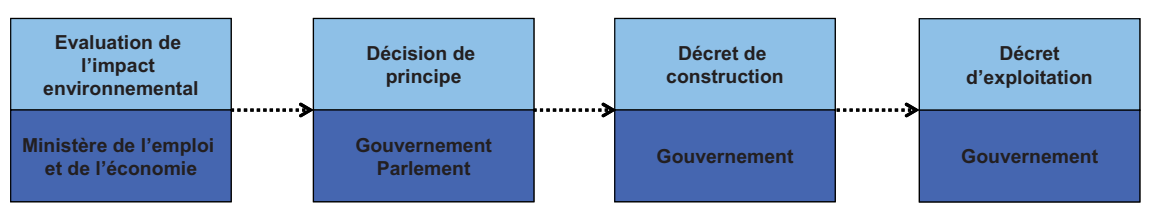

Figure 6 - Processus d'autorisation pour OL3.

Licensing process of OL3. 
l'emploi et de l'économie. Un avis positif du STUK sur la sûreté est indispensable pour que le gouvernement délivre l'autorisation de construction. Cette dernière a été délivrée en 2005 par le gouvernement. La publication, par le gouvernement, du décret d'exploitation, valable pour une durée limitée de 10 ans, est également dépendante de l'avis du STUK concernant l'instruction technique relative à l'autorisation d'exploitation. Une réévaluation de la sûreté de l'installation est, comme en France, à prévoir périodiquement.

L'évaluation de la sûreté se base sur l'avis du STUK lors de l'instruction du dossier de l'exploitant, sur le contrôle de la qualité du travail de fabrication, de construction et sur les conclusions des inspections ayant lieu pendant la phase de construction (8 inspections ont eu lieu en 2008 ; une par an est dédiée à la radioprotection).

L'autorité finlandaise et l'autorité française coopèrent activement dans le cadre de l'évaluation de la sûreté des EPR. À ce titre, à la fin de l'année 2009, elles ont publié conjointement avec leur homologue britannique (HSE) une déclaration commune sur la conception du système de contrôle-commande du réacteur EPR. Selon cette déclaration, la démarche engagée permet la promotion «d'une compréhension et une mise en œuvre partagées des standards internationaux existants, l'harmonisation de standards réglementaires et la conception de réacteurs avec le plus haut niveau de sûreté ».

\section{Prise en compte de la radioprotection à la conception}

Des différences importantes sont à noter dans le pilotage des projets. TVO n'exerce pas de rôle d'architecte industriel pour la construction de l'EPR OL3 et commande une centrale « clé en main » à AREVA alors qu'EDF, qui considère la construction de l'EPR FLA3 comme une tête de série, se positionne dans ce rôle d'architecte industriel de l'ensemble du projet. Pour l'EPR FLA3, le concepteur AREVA est responsable des études détaillées et fournisseur de la chaudière nucléaire. Il joue donc un rôle clé dans le cadre des études d'optimisation tout au long du processus de conception. C'est pourquoi, les études préliminaires de conception des deux EPR sont très proches en ce qui concerne la prise en compte de la radioprotection.

En France, comme en Finlande, la démarche d'optimisation de la radioprotection à la conception s'articule autour de la définition de cibles en termes de doses aux travailleurs et se fonde sur l'utilisation de l'expérience d'exploitation d'autres réacteurs. Les EPR profitent ainsi de technologies issues du palier N4 français et des réacteurs Konvoi allemands. 


\subsection{Détermination de l'objectif de dose et démarche d'optimisation en France}

EDF a mis en place un processus d'optimisation de la radioprotection sur la base de l'expérience acquise sur les réacteurs en exploitation.

À l'origine, dans l'édition 2003 du Preliminary Safety Analysis Report de l'EPR, EDF s'était fixé un objectif de dose collective maximale de 500 H.mSv par an (fondé sur une analyse des doses reçues, dans les réacteurs de type $1300 \mathrm{MWe}$ français et Konvoi allemands ; l'évaluation réalisée par EDF donnait une dose collective hors bâtiment réacteur, tranche en fonctionnement, d'environ $230 \mathrm{H} . \mathrm{mSv} /$ an et dans le bâtiment réacteur, en arrêt de tranche, d'environ 250 H.mSv), un objectif de dose individuelle maximale de $6 \mathrm{mSv}$ lors d'un arrêt de tranche et une valeur maximale de dose interne par inhalation de $0,2 \mathrm{mSv}$ pendant les 60 heures d'intervention prévues dans le bâtiment réacteur pendant le fonctionnement en puissance. Lors de la réunion du groupe permanent d'experts pour les réacteurs nucléaires (GPR) de juillet 2004, l'IRSN a considéré qu'EDF devrait se fixer un objectif de dose collective sur EPR plus ambitieux que la dose collective reçue sur les meilleurs réacteurs français en exploitation. Concernant l'objectif de dose individuelle, l'IRSN a considéré qu'EDF n'avait pas apporté de justification suffisante d'une démarche visant à la réduction de cette dose individuelle. Enfin, l'IRSN a considéré qu'EDF devait se fixer un objectif de dose interne nulle lors des interventions dans le bâtiment réacteur.

À la suite de la réunion du GPR, EDF a considéré que l'optimisation de la dose individuelle serait prise en considération de façon implicite en optimisant la dose des travailleurs les plus exposés, en intégrant ce critère dans le choix des activités à enjeux radioprotection à optimiser. EDF a approfondi sa démarche d'analyse de l'expérience des réacteurs en exploitation dans le domaine de la radioprotection des travailleurs, en collectant les données dosimétriques des meilleurs réacteurs, correspondant aux 16 tranches des paliers P' 4 et N4 ${ }^{11}$, entre 2001 et 2003 et en prenant en considération le type d'arrêt de tranche $\left(\mathrm{ASR}^{12}, \mathrm{VP}^{13}, \mathrm{VD}^{14}\right)$ et les doses reçues hors arrêt de tranche. Sur cette base, EDF a défini une valeur de dose de référence établie à partir d'une dose moyenne ajustée au cadencement des arrêts de tranche prévus sur EPR sur 10 ans (VP-ASR-VP-ASR-VP-VD, avec des cycles de 18 mois) et en tenant compte de la dose collective moyenne annuelle de la «meilleure » tranche du parc en exploitation, à savoir Golfech 2 (sur la période

\footnotetext{
${ }^{11}$ Dernières tranches construites sur le parc (1300 et $\left.1450 \mathrm{MWe}\right)$

12 ASR : arrêt pour simple rechargement du combustible.

13 VP : arrêt pour maintenance ou visite partielle consacrée au rechargement du combustible mais aussi à un important programme périodique de maintenance.

${ }^{14} \mathrm{VD}$ : visite décennale incluant des contrôles approfondis et réglementaires des principaux composants (la cuve du réacteur, le circuit primaire et l'enceinte du bâtiment réacteur).
} 
allant de 1994 à 2003, avec un cadencement réel de 12 et 18 mois selon l'exploitation de la tranche ${ }^{15}$ ). La dose de référence ainsi évaluée s'établit à 440 H.mSv/an.

Le processus d'optimisation mis en place ensuite par EDF s'intéresse particulièrement à sept activités à enjeux radiologiques prioritaires sélectionnées à partir de critères de dose collective, de débit de dose et de propreté radiologique : activités liées aux calorifuges, aux travaux d'ouverture/fermeture de cuve, à la préparation des contrôles des générateurs de vapeur côtés primaire et secondaire, à la robinetterie RCP, RCV, RIS et $\mathrm{RRA}^{16}$, aux interventions relatives au combustible, à la logistique et au conditionnement des déchets. Ces 7 activités représentent en général en moyenne plus de $50 \%$ de la dose collective annuelle sur les réacteurs en fonctionnement.

Le processus d'optimisation est itératif et permet à chaque étape de définir des prévisions de dose intégrant les différentes options d'optimisation et évolutions de conception par rapport aux réacteurs en exploitation. Ce processus d'optimisation et les principales évolutions de conception sont décrits de manière détaillée dans l'article Minière et al. (2008). La figure 7 illustre les différentes étapes du processus décrites ci-après.

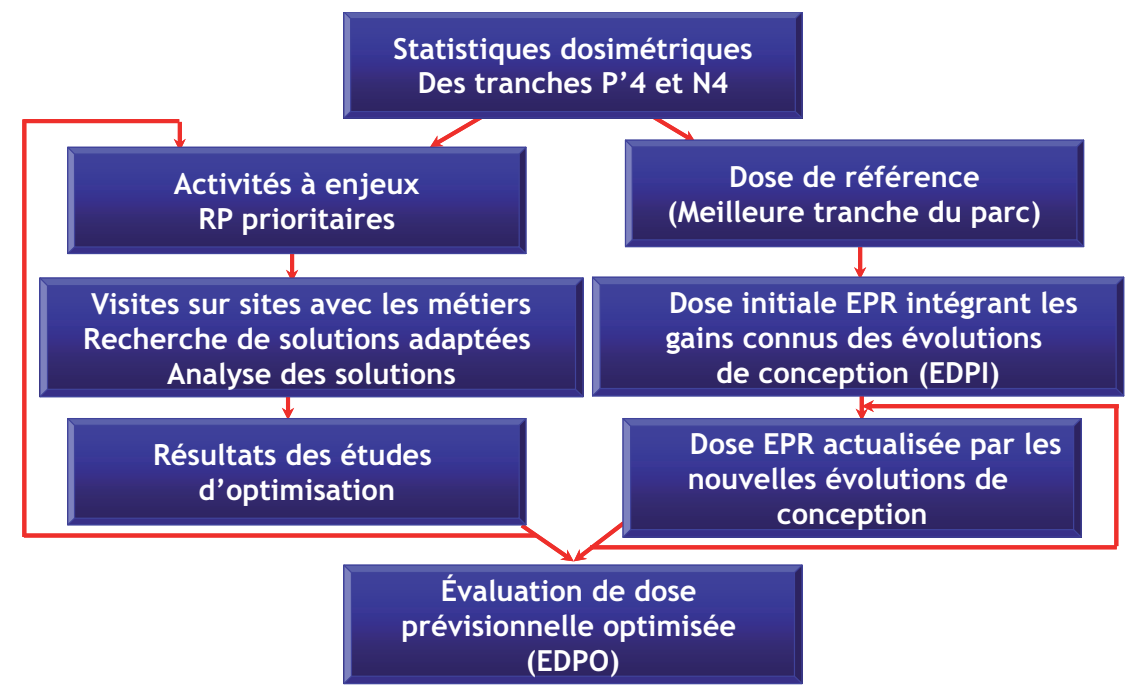

Figure 7 - Processus d'optimisation implanté par EDF.

Optimization process implemented by EDF.

\footnotetext{
15 La visite complète Initiale n'a pas été prise en compte.

${ }^{16} \mathrm{RCP}$ : circuit primaire ; RCV : circuit de contrôle volumétrique et chimique ; RIS : système d'injection de sécurité ; RRA : système de refroidissement à l'arrêt.
} 
Ainsi, dans un premier temps, l'évaluation de dose prévisionnelle initiale (EDPI) pour l'EPR est établie en se fondant sur la dose de référence et en intégrant les gains ou les pertes dosimétriques connus des «évolutions de conception ${ }^{17}$ » de l'EPR, par exemple la réduction de la quantité de stellites ${ }^{18}$ en comparaison avec les tranches en exploitation (gain de l'ordre de $15 \%$ sur toutes les activités), la mise en place d'un plancher au niveau du dôme du pressuriseur (réduction du débit de dose d'environ $75 \%$ pour les travaux réalisés dans cette zone). Cette évaluation prévisionnelle de dose initiale est établie à $361 \mathrm{H} . \mathrm{mSv} / \mathrm{an}$ (estimation de juin 2008).

Dans un deuxième temps, l'évaluation de dose prévisionnelle actualisée pour l'EPR est établie en se fondant sur l'EDPI et en prenant en considération les «nouvelles évolutions de conception ${ }^{19}$ » (par exemple l'utilisation de vannes nécessitant peu de maintenance, la mise en œuvre d'une salle de surveillance dédiée à la radioprotection...). L'évaluation de la dose prévisionnelle actualisée conduit à une valeur de 345 H.mSv/an (estimation de juin 2008).

Finalement, une évaluation de dose prévisionnelle optimisée (EDPO) est établie en se fondant sur l'évaluation de dose prévisionnelle actualisée et en tenant compte des options d'optimisation finales (pour certaines toujours en cours d'étude). L'EDPO est évaluée à $331 \mathrm{H} . \mathrm{mSv} / \mathrm{an}$ (estimation en juin 2008). La figure 8 présente la répartition, par famille d'activités, de cette évaluation de dose collective prévisionnelle optimisée. Il est à noter que l'estimation des gains associés à l'activité de conditionnement des déchets n'a pas encore été complètement évaluée par l'exploitant. L'analyse de la démarche et des notes finales d'optimisation associées fait toujours l'objet d'une instruction de la part de l'ASN et de l'IRSN.
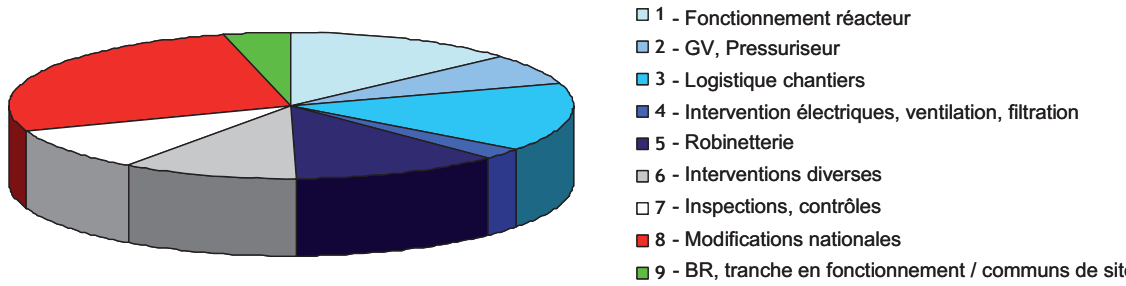

Figure 8 - Détail de la dose prévisionnelle optimisée finale pour FLA3. Detailed final target dose for FLA3.

\footnotetext{
17 Les « évolutions de conception » sont des options techniques connues.

18 Stellite : alliage métallique dur comportant du cobalt équipant classiquement la robinetterie. L'érosion de cet alliage entraîne la création de particules métalliques qui, après activation sous flux neutronique, deviennent très irradiantes. Le terme stellite est une marque de commerce.

${ }^{19}$ Les « nouvelles évolutions de conception » sont des options techniques envisagées qui nécessitent des études de faisabilité.
} 
Étant donné le caractère itératif du processus d'optimisation, les valeurs de doses prévisionnelles présentées par EDF sont encore potentiellement amenées à diminuer (pour exemple, l'EDPO était évaluée à 353 H.mSv/an en juin 2005).

L'objectif de dose collective inscrit dans le rapport de sûreté de l'EPR FLA3 reste actuellement le même qu'en 2005, soit $350 \mathrm{H} . \mathrm{mSv} / \mathrm{an}$ (cette valeur est également reportée dans le graphe de la Fig. 10). EDF a cependant indiqué dès 2005, au cours des réunions techniques, sa volonté d'essayer d'atteindre un objectif de 300 H.mSv à l'issue des études d'optimisation.

EDF doit achever, en 2010, la troisième et dernière itération du processus d'optimisation. L'IRSN a en charge l'évaluation de l'ensemble des étapes du processus itératif mis en œuvre par EDF.

Une des spécificités techniques nouvelles les plus importantes du projet EPR par rapport aux réacteurs du parc français est le concept «two-rooms» : cette évolution du design consiste à permettre des interventions du personnel dans le bâtiment réacteur en dehors des arrêts de tranche, notamment pour préparer ces derniers. Le bâtiment réacteur est séparé en deux zones bien distinctes : tranche en fonctionnement, une zone interdite, classée rouge, abritant les éléments du circuit primaire et une zone accessible, classée verte, correspondant aux espaces annulaires à tous les niveaux. Dans la zone accessible, le débit de dose « toutes composantes de rayonnements confondues » doit être inférieur à $25 \mu \mathrm{Sv} \cdot \mathrm{h}^{-1}$ et le débit de dose dû aux neutrons inférieur à $2,5 \mu \mathrm{Sv} \cdot \mathrm{h}^{-1}$. EDF envisage la possibilité d'autoriser l'entrée dans le bâtiment réacteur 7 jours avant l'arrêt de tranche et 3 jours après le redémarrage. La vérification du dimensionnement des protections permettant l'entrée dans le bâtiment réacteur, tranche en fonctionnement, fait actuellement l'objet d'une instruction de la part de l'IRSN ainsi que l'analyse de l'optimisation des activités à réaliser «tranche en fonctionnement ».

Par ailleurs, le projet EVEREST, c'est-à-dire l'accès en tenue de travail en zone contrôlée, actuellement déployé sur quelques sites du parc, notamment Golfech et Civaux, est retenu sur l'EPR de Flamanville au titre des dispositions de conception, y compris pour les accès dans le bâtiment réacteur. Ce sujet fait d'ailleurs l'objet d'un suivi de la part de l'ASN et l'IRSN.

\subsection{Détermination de l'objectif de dose et démarche d'optimisation en Finlande}

L'objectif de dose initial a été déterminé à l'origine à l'aide des critères donnés dans le guide YVL 7.18 (YVL, 2003) du STUK sur « les aspects de radioprotection 
et de sûreté nucléaire dans la conception d'une centrale nucléaire » ainsi que dans le guide « European Utility Requirements (EUR) » pour EPR :

- critère YVL $7.18:<0,5 \mathrm{H} . \mathrm{Sv} / 1 \mathrm{GWe}$ en moyenne sur la vie de l'installation,

- pour OL3 : <0,8 H.Sv /an (1600 MWe),

- critère EUR : 0,5 H.Sv /an en moyenne sur la vie de l'installation.

Sur la base de ces critères, d'une durée d'exploitation de 60 ans et de l'évaluation prévisionnelle des doses, l'objectif de dose du rapport préliminaire de sûreté de l'EPR OL3 était établi en 2006 à 425 H.mSv/an pour des cycles de 12 mois (valeur conforme aux prescriptions du guide YVL 7.18). Le détail de l'estimation de l'objectif de dose est donné sur la figure 9 en fonction de différentes familles d'activités. Les interventions menées en arrêt de tranche représentent $245 \mathrm{H} . \mathrm{mSv} / \mathrm{an}$, celles réalisées tranche en fonctionnement $30 \mathrm{H} . \mathrm{mSv} /$ an et l'estimation de la dose reçue dans les autres bâtiments que le bâtiment réacteur est de $150 \mathrm{H} . \mathrm{mSv} / \mathrm{an}$. Il est à noter que ces valeurs permettent de distinguer clairement la contribution des activités effectuées dans le bâtiment réacteur de celles réalisées dans les autres bâtiments. Cette distinction n'est, pour l'instant, pas fournie dans l'estimation de l'objectif de dose de l'EPR français ( $c f$. Sect. 3.1).

L'évaluation prévisionnelle de dose collective a depuis été mise à jour par les valeurs suivantes ${ }^{20}$ :

- 0,235 H.mSv par arrêt de tranche pour des cycles de 24 mois ;

- 0,280 H.mSv par arrêt de tranche pour des cycles de 12 mois.

Dans l'attente de la mise à jour complète de l'objectif de dose collective, c'est la valeur de 425 H.mSv/an qui est reportée dans le graphe de la figure 10.

Sur l'EPR finlandais, tout comme sur l'EPR français, une des options techniques les plus importantes mise en œuvre pour réduire la dose collective est
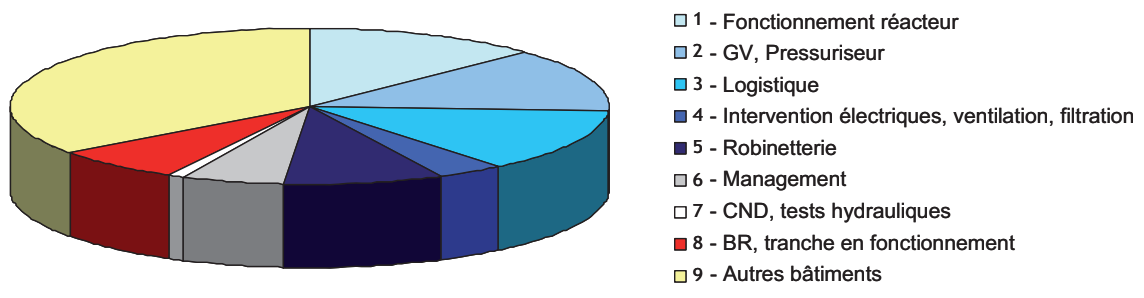

Figure 9 - Détail de l'estimation de l'objectif de dose préliminaire pour OL3. Detailed preliminary collective dose estimations for OL3.

${ }^{20}$ Données 2010 du STUK. 
la diminution de la quantité de stellites. À titre indicatif, la surface totale de stellites envisagée sur les EPR finlandais et français peut varier d'environ $4,4 \mathrm{~m}^{2}$ à $2,3 \mathrm{~m}^{2}$ suivant les stratégies retenues; cette surface est d'environ $7,2 \mathrm{~m}^{2}$ sur un réacteur français du palier $\mathrm{P}^{\prime} 4^{21}$.

Les éléments disponibles concernant la méthode d'optimisation et les options techniques envisagées sont actuellement moins détaillés par rapport à ce que peut présenter EDF pour l'EPR français. Cependant, la démarche d'optimisation finlandaise est une démarche globale qui a également intégré l'identification de zones où la réduction du débit de dose peut avoir un impact significatif sur la dosimétrie. La nature et les épaisseurs de protection biologiques ont ainsi été optimisées afin de respecter des critères maximaux de débit de dose dans des zones spécifiques, à savoir $10 \mu \mathrm{Sv} \cdot \mathrm{h}^{-1}$ à la surface de la piscine, $7,5 \mu \mathrm{Sv} \cdot \mathrm{h}^{-1}$ dans des zones de passage ou $1 \mu \mathrm{Sv} \cdot \mathrm{h}^{-1}$ pour la contribution due aux neutrons dans les zones accessibles du bâtiment réacteur pendant le fonctionnement du réacteur.

Les échanges prévus en 2010 entre le STUK, l'ASN et l'IRSN doivent permettre de faire un point sur la méthode et les options techniques retenues dans le cadre des démarches d'optimisation à la conception des réacteurs EPR français et finlandais.

\section{Comparaison des objectifs de dose associés aux EPR aux doses reçues sur d'autres réacteurs à eau sous pression}

La figure 10 présente, pour la période de 1988 à 2008, une comparaison des doses collectives annuelles des réacteurs à eau sous pression français et des objectifs de dose définis pour les EPR français et finlandais. La dose collective annuelle actuellement reçue sur les réacteurs de type Konvoi de dernière génération en Allemagne est également fournie (source : $\mathrm{ISOE}^{22}$ ).

Les objectifs de dose des EPR français et finlandais sont d'un ordre de grandeur similaire aux doses moyennées reçues sur les dernières tranches construites sur le parc français (palier N4 et Golfech 2, dernière tranche construite sur le palier $1300 \mathrm{MWe}$ ) et sont donc inférieurs aux doses collectives moyennes annuelles reçues sur les paliers 900 et 1300 MWe. Les objectifs de dose des EPR français et finlandais sont cependant supérieurs aux doses collectives annuelles reçues en moyenne dans les réacteurs Konvoi de dernière génération.

${ }^{21}$ Données du STUK de 2006 confirmant les données des études produites en 2003.

22 Les valeurs utilisées ont été extraites de la base de données ISOE. Le système ISOE (système d'information sur l'exposition professionnelle) est fondé sur un réseau de contacts et met en œuvre une base de données internationale relative à la dosimétrie et à la radioprotection dans les réacteurs électronucléaires. 


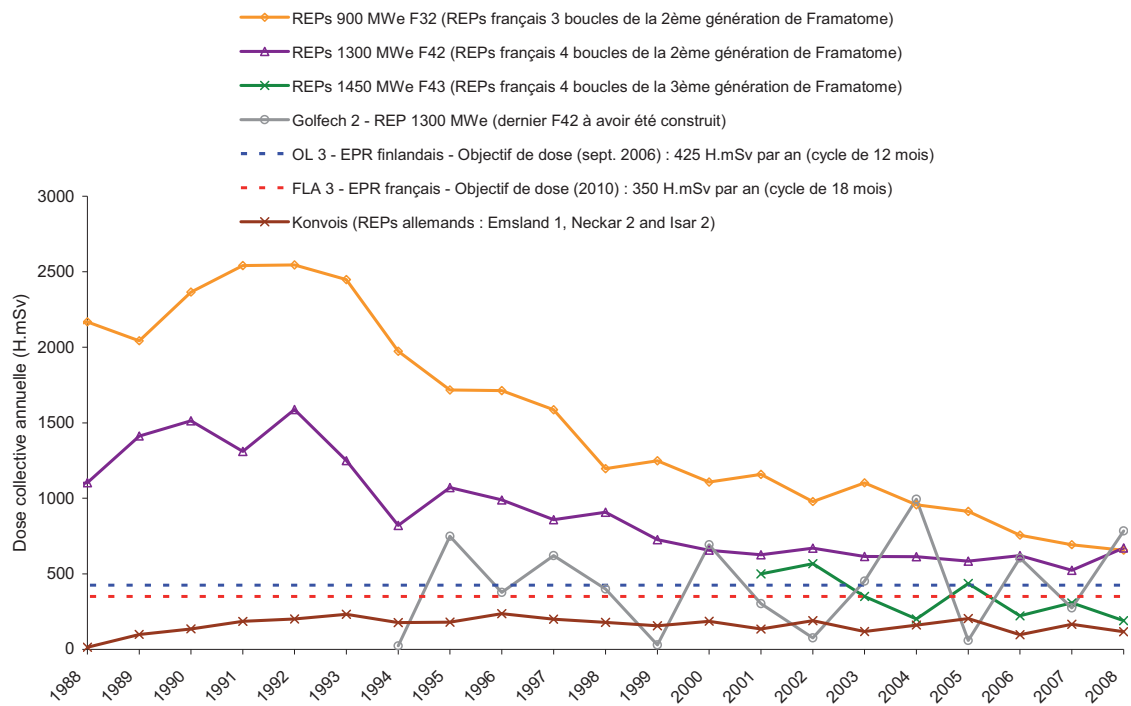

Figure 10 - Doses collectives annuelles des REPs français et allemands entre 1988 et 2008 comparées aux objectifs de dose des EPR français et finlandais (source ISOE).

Annual collective dose for French and German PWR between 1988 and 2008 compared to the EPRs targets (source ISOE).

La comparaison directe est en réalité difficile à réaliser car les valeurs de doses dépendent des choix de conception, de la durée d'un cycle, des temps de maintenance, de la durée des arrêts de tranche, des travaux à réaliser...

Ainsi, il existe des différences importantes dans la conception des réacteurs pouvant expliquer une partie des écarts entre les valeurs de dose collective annuelle reçues ou susceptibles d'être reçues sur les différents types de réacteurs. La diminution du volume de stellites est par exemple un critère fondamental dans la réduction de la dose collective. Pour les réacteurs de type Konvoi, les alliages contenant du cobalt ne sont pas utilisés pour les circuits de refroidissement du circuit primaire ni pour les systèmes directement liés au circuit primaire hors mécanismes de commande de grappes (par exemple, les vannes du circuit primaire ne contiennent pas de stellites). À titre indicatif, la surface totale de stellites admise dans un réacteur Konvoi est de 2,6 $\mathrm{m}^{2}$ ( $c f$. Sect. 3.2). La politique mise en ouvre sur les réacteurs Konvoi est également menée par EDF pour l'EPR français dans la mesure du possible pour réduire le nombre de robinets ou de clapets contenant des portées stellitées (cette démarche est bien sûr également menée pour l'EPR finlandais). EDF envisage notamment de mettre en œuvre des robinets à 
maintenance allégée, des robinets à démontage ultra rapide et des clapets à maintenance allégée sans stellite sur l'EPR. Parallèlement aux études menées pour l'EPR, EDF envisage d'ailleurs également de remplacer une partie de la robinetterie existante sur le parc par ces équipements sans stellite.

Un certain nombre d'autres dispositions favorisent également la réduction de la dose sur les réacteurs français les plus récents, les EPR et les réacteurs de type Konvoi (Baumann et al., 2006) :

- l'accessibilité facilitée des organes nécessitant une maintenance périodique,

- la sectorisation du bâtiment réacteur dans le but d'isoler les différents équipements contribuant majoritairement à la dose et de limiter au maximum les doses résultant des équipements adjacents lors d'une intervention sur un équipement donné,

- la mise en œuvre de protections biologiques permanentes ou amovibles,

- la réduction des opérations de maintenance (mise en place d'équipements nécessitant le minimum de maintenance, réduction importante du nombre de soudures à contrôler en arrêt de tranche au niveau du générateur de vapeur par exemple, possibilité d'effectuer certaines opérations de maintenance dans des zones de plus faible débit de dose...),

- la possibilité de décontamination de certains équipements avant les opérations de maintenance (grandes cuves par exemple, mise en place permanente d'équipements de décontamination pour certaines opérations),

- l'aménagement d'espaces de travail dans des zones où le débit de dose est faible,

- la télémanipulation pour certaines interventions (par exemple le contrôle des soudures au niveau du circuit primaire et du générateur de vapeur).

La mise en œuvre de ces dispositions influe significativement sur les gains potentiels en termes de doses aux travailleurs et diffère d'un type de réacteur à l'autre.

En ce qui concerne la durée des cycles, elle est en moyenne de 12 mois sur les paliers $900 \mathrm{MWe}$ français et de l'ordre de 16 mois sur les autres réacteurs du parc français. Les objectifs de dose sont évalués pour des cycles de 18 mois pour l'EPR français et de 12 mois pour l'EPR finlandais.

Enfin, pour ce qui est de l'impact de la maintenance et des arrêts de tranche sur la dosimétrie des travailleurs et afin d'éclairer les comparaisons effectuées en se fondant sur la figure 10, les durées moyennes des arrêts de tranche sont données sur la figure 11 pour les réacteurs français en exploitation les plus récents (paliers P'4 et N4) et les réacteurs de type Konvoi de dernière génération pour la période de 1988 à 2008. On constate que les durées associées aux arrêts de tranche (temps 


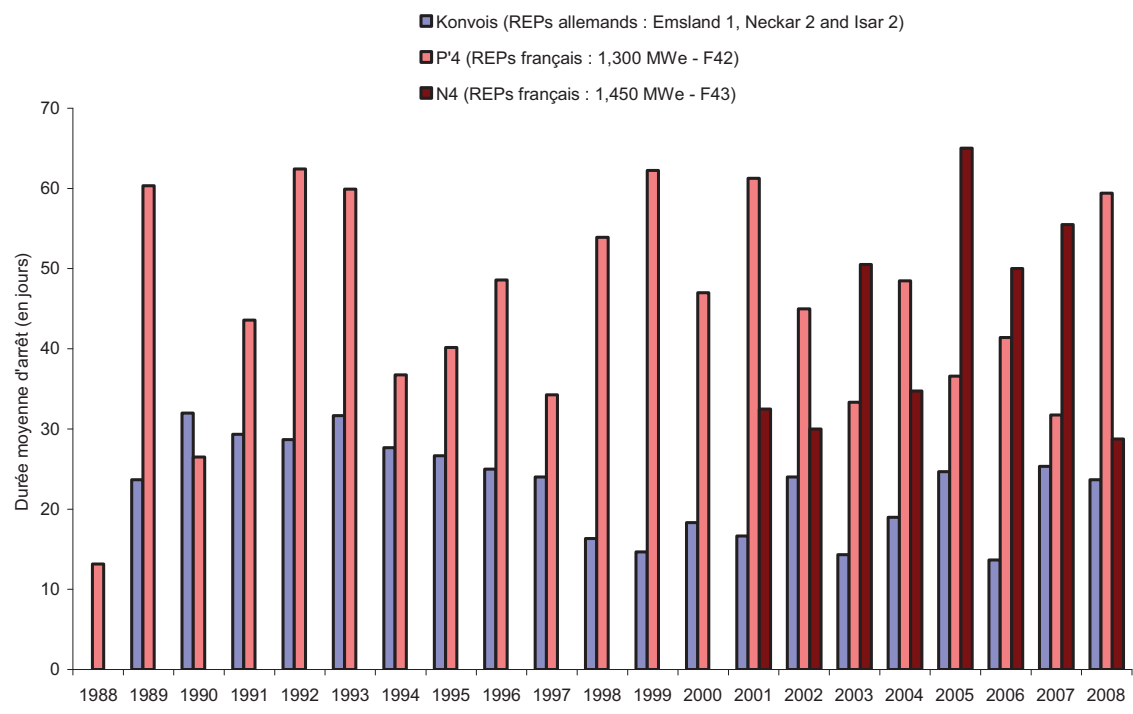

Figure 11 - Évolution de la durée moyenne d'arrêt de tranche pour les réacteurs français de type P'4 et $\mathrm{N} 4$ et les dernières générations de réacteurs de type Konvoi entre 1988 et 2008 (source : ISOE). Evolution of mean outage duration for P'4 and N4 French PWR between 1988 and 2008 compared to the last generation of Konvois (source: ISOE).

de maintenance) sont, en moyenne, deux fois plus importants pour les réacteurs français que pour les réacteurs Konvoi. Il faut aussi évidemment considérer que les réacteurs de type Konvoi bénéficient d'un design très différent des réacteurs actuellement exploités sur le parc français (piscine combustible à l'intérieur du bâtiment réacteur, etc.). Une partie de la maintenance peut-être réalisée «tranche en fonctionnement » sur les réacteurs Konvoi de la même façon que cela est prévu sur l'EPR.

Cependant, de manière générale, pour les réacteurs de type Konvoi comme pour les réacteurs français actuellement en exploitation, plus des trois quarts de la dose collective annuelle sont reçus lors des arrêts de tranche ${ }^{23}$. Donc, si les temps de maintenance pour l'EPR français étaient du même ordre de grandeur que pour les réacteurs français en exploitation, l'écart constaté entre l'objectif de dose de l'EPR français et les doses collectives annuelles reçues en moyenne dans les réacteurs Konvoi de dernière génération s'expliquerait en grande partie par des durées d'arrêt plus courtes sur les réacteurs Konvoi.

\footnotetext{
23 À l'exception de Neckar 2, pour lequel l'expérience d'exploitation est la plus faible et peu représentative.
} 
Il s'avère toutefois que les objectifs de durée d'arrêts de tranche pour l'EPR français sont inférieurs aux durées d'arrêts du parc ; ces objectifs sont les suivants (Guehl et Loutouby, 2009) :

- inférieur à 11 jours pour un ASR ;

- inférieur à 16 jours pour une VP ;

- inférieur à 40 jours pour une VD.

L'écart constaté entre l'objectif de dose de l'EPR français et les doses collectives annuelles reçues en moyenne dans les réacteurs Konvoi de dernière génération ne s'explique donc pas aussi simplement.

Enfin, dans le cadre des études de conception, il est utile de rappeler qu'un certain nombre de «marges» sont conservées. L'importance relative de ces marges sur les EPR devrait contribuer très certainement à une dose collective annuelle effectivement reçue inférieure aux objectifs de dose actuellement définis.

\section{Conclusion}

Alors que la construction de deux EPR est actuellement en cours en Europe (en France et en Finlande), la comparaison entre les deux systèmes d'instruction français et finlandais ainsi qu'entre les démarches d'optimisation mises en œuvre par les deux maîtres d'ouvrages et exploitants permet de cerner les points communs et les différences associées à la mise en œuvre de la radioprotection et de son contrôle en phase de conception.

Les études d'optimisation étant encore en cours, les objectifs de dose définis aujourd'hui pour les deux EPR sont probablement amenés à diminuer. Il convient également d'ajouter que les objectifs de dose définis à la conception d'un réacteur reposent sur des estimatifs dosimétriques qui comportent nécessairement des marges et que les doses réellement reçues seront certainement sensiblement inférieures à ces objectifs. En l'état actuel, les objectifs de dose définis pour l'EPR de Flamanville permettent d'envisager des doses reçues lors de l'exploitation inférieures à celles actuellement reçues en moyenne sur les réacteurs français.

Outre les deux EPR actuellement en construction en Europe, deux autres réacteurs de type EPR sont également en construction à Taïshan en Chine et un second EPR français doit être construit sur le site de Penly, en Seine-Maritime (la demande d'autorisation de construction est prévue d'être déposée en octobre 2010). L'EPR est également en cours de certification par la NRC aux États-Unis et d'autres pays s'intéressent à cette technologie de réacteurs pour agrandir ou renouveler leurs parcs nucléaires actuels. 
Enfin, un groupe de travail (dans le cadre des travaux coordonnés par l'Agence pour l'énergie nucléaire: Multinational Design Evaluation Program - EPR Working Group), regroupant des représentants des autorités de sûreté du Canada, de Chine, de Finlande, de France, du Royaume-Uni et des États-Unis, s'intéresse actuellement aux nouveaux designs de réacteurs et en particulier à l'EPR. Les aspects liés à la radioprotection feront notamment l'objet d'échanges sur les positions techniques des différentes autorités de sûreté. L'IRSN participera à ces travaux.

\section{RÉFÉRENCES}

Baumann E. et al. (2006) The EPR: A clear step forward in dose reduction and radiation protection, Nucl. Engineer. Des. 236, 1720-1727.

Bouchez E., Couasnon O., Evrard J.-M., Riihiluoma V., Beneteau Y., Foret J.-L. (2009) EPR Présentation comparée des instructions Françaises et Finlandaises et des démarches d'optimisation de la radioprotection à la conception, In Congrès National de Radioprotection SFRP, 16-18 juin, 2009, Angers.

Couasnon O., Bouchez E., Gourram H., Evrard J.-M., Riihiluoma V., Beneteau Y., Foret J.-L. (2008) Comparative approach of the French and Finnish reviewing process and optimization of radiation protection at the design phase, In 12th International Congress of the International Radiation Protection Association, 19-24 october, 2008, Buenos Aires, Argentina.

DSIN (1993) Lettre DSIN/Paris n 1321/93 du 22 juillet 1993 : objectifs généraux retenus pour la prochaine génération de réacteurs à eau sous pression.

Gourram H., Couasnon O., Evrard J.-M., Riihiluoma V., Beneteau Y., Foret J.-L. (2008) Comparative approach of the French and Finnish instructions and optimization of radiation protection at the design phase, In 2008 ISOE International Symposium, June, 2008, Turku, Finland.

Guehl J.-C., Loutouby A. (2009) Évolution de l'exploitation et de la maintenance de génération 2 à génération 3 - EPR, Congrès SFEN «Evolution de l'exploitation et de la maintenance des réacteurs », décembre 2009, Paris, France.

Minière D. et al. (2008) Les enjeux radioprotection de l'EPR, Radioprotection 43, 273-287.

YVL (2003) Guide YVL 7.18 Radiation safety aspects in the design of a nuclear power plant, STUK, 26 september 2003 